\title{
Impacto de las nuevas definiciones en la prevalencia del síndrome metabólico en una población adulta de Bucaramanga, Colombia
}

\author{
Juan Bernardo Pinzón, Norma Cecilia Serrano, Luis Alfonso Díaz, Gerardo Mantilla, \\ Harvey Mauricio Velasco, Luz Ximena Martínez, Paula Andrea Millán, \\ Sandra Milena Acevedo, Daniel Moreno
}

Centro de Investigaciones Biomédicas, Facultad de Medicina, Universidad Autónoma de Bucaramanga, Bucaramanga, Colombia

Introducción. La prevalencia de síndrome metabólico depende de los criterios de clasificación empleados, como son los de la International Diabetes Federation y el Adult Treatment Panel o su modificación.

Objetivo. Comparar la prevalencia del síndrome metabólico generada por cada una de las tres definiciones en una población de adultos.

Materiales y métodos. Se estudiaron 155 personas (54,2\% varones, edad promedio de 40,9 años). Se aplicaron las tres definiciones y se comparó la prevalencia con la prueba de Wilcoxon y la concordancia con kappa de Cohen.

Resultados. La prevalencia de síndrome metabólico según el Adult Treatment Panel-III (ATPIII) fue 12,3\% (IC95\% 7,5-18,5), según el Adult Treatment Panel-IIla (ATP-IIIa), 34,8\% (IC95\% 27,4-42,9) y según la International Diabetes Federation (IDF), 32,9\% (IC95\% 25,6-40,9). La prevalencia por ATP-III es inferior a la vista por ATP-IIla e IDF $(p<0,001)$, pero entre estas dos últimas es similar $(p=0,083)$. Hay pobre concordancia entre ATP-III y ATP-IIIa $(\kappa=0,414$, IC95\% $0,409-0,420)$, y entre ATP-III e IDF ( $\kappa=0,374$, IC95\% 0,368-0,379), pero muy buena entre ATPIlla e IDF ( $\kappa=0,957$, IC95\% 0,950-0,963).

Conclusión. Las nuevas definiciones para el síndrome metabólico (ATP-IIla e IDF) aumentan tres veces la prevalencia del diagnóstico, sin encontrar diferencias entre ellas, a pesar de que la última organización incluye la obesidad central como un criterio necesario para el diagnóstico, cuando en la primera no se hace igual ponderación.

Palabras clave: coronariopatía, resistencia a la insulina, obesidad, diagnóstico.

Impact of the new definitions in the prevalence of the metabolic syndrome in an adult population at Bucaramanga, Colombia

Introduction. The prevalence of metabolic syndrome depends on the criteria used for its classification. Three criteria in common use are those from International Diabetes Federation (IDF), the Adult Treatment Panel (ATP-III) or its update (ATP-IIIa).

Objective. The prevalence statistic for generated for metabolic syndrome was compared for on the basis of each of the three criteria.

Materials and methods. The sample consisted of 155 teachers and employees in the school of medicine. The average age was $40.9 ; 54.2 \%$ were men. The three criteria were applied and the prevalences were compared with the Wilcoxon test and Cohen's kappa.

Results. Metabolic syndrome prevalence generated by each criterion was as follows: ATP-III was $12.3 \%(95 \% \mathrm{Cl} 7.5-18.5)$, ATP-IIla was $34.8 \%(95 \% \mathrm{Cl} 27.4-42.9)$ and IDF $32.9 \%(95 \% \mathrm{Cl}$ 25.6--40.9). The prevalence indicated by ATP-III was lower than the ATP-IIla or IDF prevalances $(p<0,001)$; however those of ATPIII-a and IDF were similar $(p=0,083)$. Poor agreement was seen between ATP-III and ATP-IIIa ( $\mathrm{k}=0.414$, IC95\% 0.409-0.420), and between ATP-III and IDF ( $k=0.374$, IC95\% 0.368-0.379); however, very good agreement was obtained between ATP-IIla and IDF ( $\mathrm{k}=0.957, \mathrm{IC} 95 \%$ 0.950-0.963). 
Conclusion. The new definitions for metabolic syndrome, ATP-IIla and IDF, increase the prevalence statistic by three times. This occurred despite the inclusion in IDF of an obesity factor in the criteria set.

Key words: coronary disease, insulin resistance, obesity, diagnosis.

Los países latinoamericanos afrontan una epidemia de enfermedad arterial coronaria; en 1.990 los trastornos cardiovasculares fueron la principal causa de muerte en Latinoamérica: causaron 789.000 muertes, en comparación con 473.000 muertes por enfermedades parasitarias e infecciosas $(1,2)$. En Colombia se observó un incremento en la tasa de mortalidad por enfermedad coronaria en personas de 20 a 84 años de $75,9 / 100.000$ entre 1980 y 1984 , a $97,3 / 100.000$ entre 1990 y 1996, con un incremento anual aproximado de $2,1 \%$ a través del período estudiado (3). Se espera en el futuro un incremento en la incidencia de enfermedad coronaria debido al envejecimiento de la población y a un aumento en la prevalencia de factores de riesgo cardiovascular (hipertensión, obesidad, diabetes y dislipidemia) $(2,4)$.

La constelación de algunos de estos factores de riesgo cardiovascular: obesidad abdominal, niveles altos de triglicéridos, bajos niveles de colesterol HDL, incremento en la presión arterial $y$ altos niveles de glucosa, se conocen como síndrome metabólico. El síndrome metabólico es reconocido como factor de riesgo asociado al incremento en la incidencia de diabetes, infarto agudo del miocardio y enfermedad cerebrovascular (5-7). En Colombia se ha encontrado que el riesgo de infarto del miocardio no fatal se eleva con cada uno de los componentes del síndrome metabólico de forma aislada (OR para hipertensión=2,1; diabetes $=1,87$; y obesidad abdominal=2,94) (8), lo cual permite pensar que el impacto de este síndrome debería ser, al menos, igual al observado en poblaciones similares en lo

\footnotetext{
Correspondencia:

Juan Bernardo Pinzón, Centro de Investigaciones Biomédicas, Facultad de Medicina, Universidad Autónoma de Bucaramanga, Calle 157 No. 19-55, Bucaramanga, Colombia.

Teléfono: 6399156, fax 6399147

genesis@unab.edu.co
}

Recibido: 20/06/06; aceptado: 12/02/07 étnico y en lo ambiental, como son los mexicanoamericanos o la población mexicana $(9,10)$. Actualmente, el síndrome metabólico (sin que el individuo sea diabético) se considera por sí mismo un riesgo cardiovascular intermedio, lo que predice una probabilidad entre $10 \%$ y $20 \%$ de desarrollar eventos cardiovasculares en los siguientes 10 años (11).

En países desarrollados, como Estados Unidos, la prevalencia reportada ajustada por edad del síndrome metabólico, usando los criterios del Adult Treatment Panel-III (ATP-III) (11), es de $23,7 \%$ para la población general, con una prevalencia similar en hombres $(24,0 \%)$ y en mujeres (23,4\%) (12). En Colombia, algunos resultados preliminares sugirieron que, para el período 1993 a 1996, la prevalencia en población urbana era de un 33\% (13). Así mismo, en la población de El Retiro (Antioquia), la prevalencia fue de $33,9 \%$ (ajustada por edad: $23,6 \%$ ), utilizando la definición del ATP III (13). Investigaciones realizadas en Venezuela dan a su vez cuenta de una variación en la prevalencia entre diferentes grupos raciales dentro del país: $17,0 \%$ en amerindios, $27,2 \%$ en negros, $33,3 \%$ en blancos, y $37,4 \%$ en mestizos (14).

Más allá de estas diferencias, a las cuales probablemente también somos susceptibles, no se ha realizado hasta el momento una comparación directa del impacto que las variaciones en la definición del síndrome metabólico, sobre todo luego de la modificación propuesta para la ATPIII actualizada (ATP-IIla) (15) o los criterios propuestos por la International Diabetes Federation (IDF) (16), generan en la prevalencia de esta enfermedad en nuestra población.

El objetivo de este informe es comparar la prevalencia del síndrome metabólico con base en las tres definiciones existentes, en una población de empleados (profesores y personal administrativo) de la Facultad de Medicina de la Universidad Autónoma de Bucaramanga, Colombia. 


\section{Materiales y métodos}

Esta comunicación hace parte del proyecto Génesis, un estudio piloto de naturaleza transversal, realizado entre los empleados de la Facultad de Medicina de la Universidad Autónoma de Bucaramanga, que busca identificar factores de riesgo convencionales y genéticos asociados con el síndrome metabólico y su correlación con fenotipos intermedios.

El estudio fue aprobado previamente por el Comité de Ética en Investigación de la Facultad de Medicina de la UNAB. Todos los participantes dieron su consentimiento informado por escrito. El presente artículo hace referencia particular a la prevalencia de factores de riesgo cardiovascular convencionales y del síndrome metabólico, así como las diferencias que esta última prevalencia muestra con las variadas definiciones actualmente existentes para dicho síndrome.

Todos los empleados de la Facultad de Medicina fueron invitados a participar; asistieron a una entrevista clínica, examen físico y toma de pruebas de laboratorio, a fin de establecer si se cumplían los criterios de las tres definiciones del síndrome metabólico (cuadro 1) $(11,15,16)$. No se incluyeron personas con enfermedad crónica grave (insuficiencia hepática, insuficiencia renal, cáncer, diabetes tipo 1), infecciones sistémicas o embarazo, dado que el objetivo central de la investigación era evaluar la asociación que pudiera existir entre el síndrome, los fenotipos intermedios y los polimorfismos de algunas citocinas, fenómeno que puede verse confundido por la presencia de dichas condiciones.

La entrevista clínica indagó sobre edad, sexo y antecedentes personales de enfermedad crónica o embarazo. Durante el examen físico se realizaron dos tomas de presión arterial y de perímetro de cintura $(17,18)$. Para definir el cumplimiento o no de los distintos criterios del síndrome metabólico, a todos los participantes se les realizó glucemia en ayunas, colesterol total, colesterol HDL y triglicéridos. En aquellas personas con glucemia en ayunas entre $100-125 \mathrm{mg} / \mathrm{dl}$, se les realizó una glucemia poscarga a las dos horas, para establecer si los pacientes eran, además, intolerantes a la glucosa o diabéticos tipo 2. A todas las personas se les informó por escrito los resultados de su evaluación, exhortándolos a acudir a su médico en los casos en los que se hallaron condiciones que se consideran anormales.

La glucemia se midió con el método de la glucosaoxidasa; el colesterol total por la técnica de la colesterol éster hidrolasa-colesterol oxidasa; el colesterol HDL se determinó precipitando el suero con ácido fosfotúngstico, cuantificándolo con la misma técnica del colesterol total; los triacilgliceroles se analizaron por la técnica de la glicerol fosfato-oxidasa y la glicerol cinasa.

La prevalencia del síndrome metabólico se estableció aplicando los criterios de las tres definiciones junto con su intervalo de confianza

Cuadro 1. Definiciones clínicas existentes para establecer la presencia de síndrome metabólico

\begin{tabular}{|c|c|c|}
\hline $\begin{array}{c}\text { ATP III } \\
\text { Tres o más de los siguientes criterios }\end{array}$ & $\begin{array}{c}\text { ATP III actualizada } \\
\text { Tres o más de los siguientes criterios }\end{array}$ & $\begin{array}{l}\text { IDF } \\
\text { Obesidad abdominal más dos } \\
\text { de los criterios que le siguen }\end{array}$ \\
\hline $\begin{array}{l}\text { 1. Glucemia en ayunas } \geq 110 \mathrm{mg} / \mathrm{dl} \\
\text { (incluye diabetes mellitus) } \\
\text { 2. Obesidad abdominal: } \\
\text { - Hombres }>102 \mathrm{~cm} \\
\text { - Mujeres }>88 \mathrm{~cm} \\
\text { 3. HDL bajo: } \\
\text { - Hombres }<40 \mathrm{mg} / \mathrm{dl} \\
\text { - Mujeres }<50 \mathrm{mg} / \mathrm{dl} \\
\text { 4. Presión arterial } \geq 130 / 85 \\
\text { mm Hg } \\
\text { 5. Triglicéridos } \geq 150 \mathrm{mg} / \mathrm{dl}\end{array}$ & $\begin{array}{l}\text { 1. Glucemia ayunas } \geq 100 \mathrm{mg} / \mathrm{dl} \\
\text { (incluye diabetes mellitus) } \\
\text { 2. Obesidad abdominal: } \\
\text { - Hombres } \geq 90 \mathrm{~cm} \\
\text { - Mujeres } \geq 80 \mathrm{~cm} \\
\text { 3. HDL bajo: } \\
\text { - Hombres }<40 \mathrm{mg} / \mathrm{dl} \\
\text { - Mujeres }<50 \mathrm{mg} / \mathrm{dl} \\
\text { 4. Presión arterial } \geq 130 / 85 \mathrm{~mm} \\
\text { Hg o recibir terapia antihipertensiva } \\
\text { 5. Triglicéridos } \geq 150 \mathrm{mg} / \mathrm{dl}\end{array}$ & $\begin{array}{l}\text { Obesidad abdominal: } \\
\text { - Hombres } \geq 90 \mathrm{~cm} \\
\text { - Mujeres } \geq 80 \mathrm{~cm} \\
\text { 1. Glucemia en ayunas } \geq 100 \mathrm{mg} / \mathrm{dl} \\
\text { (incluye diabetes mellitus) } \\
\text { 2. HDL bajo o tratamiento para elevarlo: } \\
\text { - Hombres }<40 \mathrm{mg} / \mathrm{dl} \\
\text { - Mujeres }<50 \mathrm{mg} / \mathrm{dl} \\
\text { 3. Presión arterial } \geq 130 / 85 \mathrm{~mm} \\
\text { Hg o recibir terapia antihipertensiva } \\
\text { 4. Triglicéridos } \geq 150 \mathrm{mg} / \mathrm{dl} \text { o recibir } \\
\text { terapia específica para reducirlos }\end{array}$ \\
\hline
\end{tabular}


del 95\% (IC95\%). Se utilizó la definición propuesta por el National Cholesterol Education Program (NCEP)/Adult Treatment Panel III (ATP III) (10), y la recientemente propuesta por la IDF (15). Para la definición actualizada del ATPIII (ATPIIla) (16) se utilizó como límite de perímetro abdominal el mismo propuesto en las guías de la IDF para suramericanos, es decir, $90 \mathrm{~cm}$ para varones $\mathrm{y}$ $80 \mathrm{~cm}$ para mujeres.

Las diferencias en la prevalencia por sexo de los factores de riesgo que condicionan el diagnóstico del síndrome metabólico se evaluaron por medio de la prueba de $\chi^{2}$. Las prevalencias resultantes de las diferentes definiciones se compararon por medio de la prueba pareada de los signos de Wilcoxon (19) y, la concordancia, por medio de la kappa media de Cohen (к) (20) y su IC95\%. Se consideraron significativas aquellas pruebas con $p<0,05$. Todos los procesos se hicieron con STATA 9.2 (21).

\section{Resultados}

De las 167 personas empleadas en la facultad, 155 aceptaron participar en el estudio; 84 eran hombres $(54,2 \%)$ y 71 mujeres $(45,8 \%)$. No fue necesario excluir a ninguno de los participantes por cuenta de enfermedad crónica grave o embarazo. La edad osciló entre 22 y 73 años, con promedio de 40,9 años (desviación estándar, $D E=10,3)$, siendo las mujeres más jóvenes que los hombres (35,5 años [DE=10,2] Vs. 44,5 años
$[D E=9,6] ; p<0,001)$. En el cuadro 2 se puede apreciar la proporción de participantes que cumplen cada uno de los criterios de dichas definiciones, tanto para la población global como por sexo. Hay diferencias entre hombres y mujeres en la prevalencia de obesidad abdominal, la presión arterial elevada y la hipertrigliceridemia.

La prevalencia según cada definición fue: ATP-III 12,3\% (IC95\% 7,5-18,5), ATP-IIIa 34,8\% (IC95\% $27,4-42,9)$ e IDF $32,9 \%$ (IC95\% 25,6-40,9). La prevalencia por ATP-III es inferior a la vista por ATP-IIla e IDF $(p<0,001)$, pero entre estas dos últimas es similar $(p=0,083)$.

Hay pobre concordancia entre ATP-III y ATP-IIla ( $\kappa=0,414$, IC95\% 0,409-0,420), y entre ATP-III e IDF ( $\kappa=0,374$, IC95\% 0,368-0,379), pero muy buena entre ATP-IIla e IDF ( $\kappa=0,957$, IC95\% 0,950-0,963), tal como se puede apreciar en el cuadro 3.

\section{Discusión}

Recientemente ha surgido la controversia sobre si el síndrome metabólico por sí mismo es una entidad clínica propia, o si este agrupamiento de factores de riesgo no provee un valor de predicción clínico más allá del que cada uno de sus componentes genera por sí mismo (22). Las principales críticas surgen de las definiciones poco precisas y muy variables actualmente vigentes, y, además, a la falta de un claro, en apariencia, sustrato patogénico. Todo esto ha

Cuadro 2. Prevalencia de factores de riesgo que condicionan el diagnóstico de síndrome metabólico.

\begin{tabular}{|c|c|c|c|c|}
\hline \multirow[b]{2}{*}{ Criterio } & \multicolumn{3}{|c|}{ Población } & \multirow[b]{2}{*}{$\begin{array}{l}\chi^{2} \\
(p)\end{array}$} \\
\hline & $\begin{array}{c}\text { Hombres } \\
(n=84)\end{array}$ & $\begin{array}{c}\text { Mujeres } \\
(n=71)\end{array}$ & $\begin{array}{c}\text { Total } \\
(n=155)\end{array}$ & \\
\hline Obesidad abdominal ( $\geq 90 \mathrm{~cm}$ hombres, $\geq 80 \mathrm{~cm}$ mujeres) & $\begin{array}{c}63 \\
(75,0 \%)\end{array}$ & $\begin{array}{c}20 \\
(28,2 \%)\end{array}$ & $\begin{array}{c}83 \\
(53,6 \%)\end{array}$ & $\begin{array}{c}33,91 \\
(<0,001)\end{array}$ \\
\hline Presión arterial elevada ( $\geq 130 / 85$ mm Hg) & $\begin{array}{c}21 \\
(25,0 \%)\end{array}$ & $\begin{array}{c}5 \\
(7,0 \%)\end{array}$ & $\begin{array}{c}26 \\
(16,8 \%)\end{array}$ & $\begin{array}{c}8,89 \\
(0,003)\end{array}$ \\
\hline Triglicéridos elevados ( $\geq 150$ mg/dL) & $\begin{array}{c}44 \\
(52,4 \%)\end{array}$ & $\begin{array}{c}7 \\
(9,9 \%)\end{array}$ & $\begin{array}{c}51 \\
(32,9 \%)\end{array}$ & $\begin{array}{c}31,51 \\
(<0,001)\end{array}$ \\
\hline Hiperglucemia ( $\geq 100 \mathrm{mg} / \mathrm{dL})$ & $\begin{array}{c}5 \\
(6,0 \%)\end{array}$ & $\begin{array}{c}1 \\
(1,4 \%)\end{array}$ & $\begin{array}{c}6 \\
(3,9 \%)\end{array}$ & $\begin{array}{c}1,09 \\
(0,297)\end{array}$ \\
\hline HDL bajo ( $<40 \mathrm{mg} / \mathrm{dL}$ varones, $<50 \mathrm{mg} / \mathrm{dL}$ mujeres) & $\begin{array}{c}80 \\
(95,2 \%)\end{array}$ & $\begin{array}{c}69 \\
(97,2 \%)\end{array}$ & $\begin{array}{c}149 \\
(96,1 \%)\end{array}$ & $\begin{array}{c}0,04 \\
(0,836)\end{array}$ \\
\hline
\end{tabular}


Cuadro 3. Concordancia entre los tres criterios utilizados para definir síndrome metabólico.

\begin{tabular}{|c|c|c|c|c|c|c|c|c|}
\hline \multirow{4}{*}{$\begin{array}{l}\text { ATPIIIa } \\
+ \\
-\end{array}$} & \multicolumn{2}{|c|}{ ATP-III } & \multirow[b]{2}{*}{ IDF } & \multicolumn{2}{|c|}{ ATP-III } & \multirow[b]{2}{*}{ IDF } & \multicolumn{2}{|c|}{ ATP-IIla } \\
\hline & + & - & & + & - & & + & - \\
\hline & 19 & 35 & + & 17 & 34 & + & 51 & 0 \\
\hline & 0 & 101 & - & 2 & 102 & - & 3 & 101 \\
\hline \multicolumn{3}{|c|}{$p<0,001$} & \multicolumn{3}{|c|}{$p<0,001$} & \multicolumn{3}{|c|}{$p=0,083$} \\
\hline \multicolumn{3}{|c|}{$\kappa=0,414$} & \multirow{2}{*}{\multicolumn{3}{|c|}{$\begin{aligned} \kappa & =0,374\end{aligned}$}} & \multirow{2}{*}{\multicolumn{3}{|c|}{$\begin{array}{c}\kappa=0,957 \\
(1 C 95 \% 0,950-0.963)\end{array}$}} \\
\hline \multicolumn{3}{|c|}{$($ IC95\% 0,409-0,420) } & & & & & & \\
\hline
\end{tabular}

generado, además de una saludable discusión dentro de la comunidad médica, un esfuerzo por caracterizar de manera más apropiada y precisa esta patología. Entre estos esfuerzos, tanto el ATP-III como la IDF han generado nuevas definiciones que permiten homogenizar los criterios existentes y preservar el valor de este diagnóstico como un marcador de riesgo cardiovascular y de diabetes.

La definición de la IDF da un valor fundamental a la presencia de obesidad abdominal y la considera el centro gravitador alrededor del cual cobran importancia el resto de factores de riesgo $(23,24)$. Así mismo, hace eco a múltiples investigaciones que determinan que los puntos de corte para obesidad visceral previamente utilizados no pueden generalizarse a todas las etnias, por lo que parece necesario que para cada una de ellas se utilice el límite a partir del cual los riesgos inherentes a este tipo de obesidad aumentan de manera sustancial. Numerosos estudios, incluso algunos realizados en nuestro país, apuntan también en la misma dirección (25).

EI ATP III, por su parte, también realizó una adaptación de sus criterios, pero no da un valor diferencial mayor a ninguno de ellos sobre los demás y, si bien reconoce la diferencia de perímetros entre diferentes poblaciones, no propone puntos de corte específicos.

Como es previsible por investigaciones previas, los cambios en la prevalencia de obesidad determinan cambios en la prevalencia del síndrome metabólico (26). Dada la propuesta de la IDF de variar por etnias el punto de corte que determina el límite de adiposidad visceral, la prevalencia esperada se incrementa en proporción similar al aumento en la prevalencia de tal variable; eso es palpable en la presente investigación, en la cual la prevalencia con estos nuevos valores es casi tres veces mayor con respecto a la definición original propuesta por el Panel de tratamiento de adultos del Programa nacional de educación en colesterol de los Estados Unidos. En la definición actualizada del ATP III, si bien la obesidad visceral no es una condición "necesaria" para el diagnóstico (como sí lo es para la IDF), la utilización de los límites de 80 y $90 \mathrm{~cm}$ para la cintura de mujeres y varones aumenta la prevalencia del síndrome en una proporción similar a la observada para la de la IDF, con una concordancia "muy buena" entre ambas definiciones.

Ashner y colaboradores (12) encontraron una prevalencia de 33\% del síndrome metabólico utilizando los criterios iniciales del ATP III; por su parte, Villegas y colaboradores en Antioquia (13), encontraron una prevalencia global del $33,9 \%$ y ajustada por edad del $23,6 \%$. Estas cifras difieren de nuestros hallazgos, $12,3 \%$ utilizando los criterios originales del ATPIII. Lo anterior puede explicarse por cuanto los estudios mencionados son de tipo población y en ellos se encuentran representados grupos de mayor edad, los cuales, están prácticamente ausentes en nuestra investigación por el tipo de población estudiada, cuyo promedio de edad es bajo comparado con los estudios mencionados.

Es sabido por todos que la prevalencia aumenta en relación con la edad de los individuos. Nuestros hallazgos, no obstante y de nuevo utilizando los criterios originales del ATPIII, se asemejan mucho a los encontrados por Mendivil y colaboradores (27) en un estudio realizado en trabajadores de la Universidad Nacional de Colombia; su prevalencia de $19,5 \%$ es similar a la nuestra, y los intervalos 
de confianza de la misma se superponen. En su investigación, el promedio de edad fue de 44,4 años, muy similar al nuestro, confirmando la importancia de la representación de todos los grupos de edad para el cálculo de prevalencias de patologías que son más frecuentes en relación con el envejecimiento. Por ello, es posible afirmar que nuestras prevalencias reflejan un escenario posiblemente más alentador que el real, pero nada de lo anterior niega el aumento que las nuevas definiciones genera en tal prevalencia.

Algunas investigaciones recientes han encontrado prevalencias similares entre las definiciones del ATPIIla y la IDF en población mexicana, $22,6 \%$ y $22,3 \%$ respectivamente, con un nivel de concordancia de 0,873 mediante el estadístico $\kappa(28)$. Cuando la definición propuesta por IDF se aplica a población que reside en los Estados Unidos, la prevalencia aumenta, particularmente en hombres mexicano-americanos (es decir, en uno de los grupos étnicos en quienes se aplica un nuevo límite de adiposidad); ambas definiciones clasifican en forma apropiada al $93,0 \%$ de los individuos con síndrome metabólico (29).

La prevalencia global, ajustada por edad, encontrada en estas investigaciones aumenta desde $34,6 \%$ con los criterios originales del ATPIII, hasta $39,1 \%$ por la IDF y $40,4 \%$ para ATPIIla (con criterios del IDF como en nuestra investigación). Estos aumentos son más sustanciales, nuevamente, en grupos étnicos en los que operan efectivamente los nuevos criterios para el diagnóstico de adiposidad central.

En nuestro estudio esta observación se mantiene; podemos apreciar cómo la prevalencia aumenta sustancialmente cuando el punto de corte que define la obesidad abdominal disminuye. Ello indudablemente mejora la sensibilidad de los criterios para el diagnóstico del síndrome metabólico en personas de nuestra etnia, que realmente lo presentan, pero simultáneamente empeora la especificidad, con lo que podría estarse diagnosticando esta entidad en un grupo de individuos que eventualmente no presentan la patología.

En una población australiana a la cual se le aplicaron los criterios propuestos por la IDF, se catalogaron como síndrome metabólico de 15\% a $20 \%$ más de individuos, incrementando la prevalencia entre 0,5 y 1,5 veces en mujeres y varones, respectivamente (30).

El análisis de la presencia del síndrome en casa persona incluye diferencias de sexo, las cuales han de tenerse en cuenta siempre, tal como se hizo en este trabajo. Ahora bien, un aspecto por desarrollar en posteriores investigaciones es si las conclusiones que en el presente estudio se plantean son iguales por sexo o grupos de edad, pues el tamaño de la presente muestra no permite hacer este tipo de subanálisis.

La IDF ha recomendado un tratamiento agresivo de los individuos con síndrome metabólico para reducir, a su vez, el riesgo de enfermedad cardiovascular y diabetes. Dado que la nueva definición aumenta en forma significativa (tanto clínica como estadísticamente) la prevalencia del síndrome (como nuestro estudio lo demostró al pasar de $12,3 \%$ al $33 \%$ con la modificación de los criterios diagnósticos generada por la IDF), es previsible que más personas estén en "riesgo" de recibir tratamiento, incluyendo medicaciones, por lo que en base a este diagnóstico per se aumenta el riesgo cardiovascular, al menos, una categoría. Esto último tendría un gran impacto para los sistemas de salud y, en especial, para economías emergentes como la nuestra. Es indudable la necesidad de estudios de costo-beneficio, en cuanto a la implementación de terapias farmacológicas precoces para estos pacientes.

No obstante esta prevalencia creciente del síndrome metabólico, ello mismo constituye una oportunidad única de prevención, mediante la implementación de programas que promueven de forma cierta los cambios terapéuticos en el estilo de vida, de indudable utilidad para cada una de las patologías que hacen parte del síndrome. Particularmente, la recomendación de planes nutricionales más balanceados y saludables, la necesidad obligatoria de información nutricional para todos los alimentos en venta y, finalmente, el reconocimiento del ejercicio como una necesidad fundamental para la salud de los individuos. Se conoce que la elevación del índice de masa corporal, el aumento en la ingestión de carbohidratos y la disminución en fibra, son 
factores de riesgo para el desarrollo de síndrome metabólico (riesgo relativo [RR] entre 1,3 y 1,9), mientras que la actividad física es protectora (RR=0,84; IC95\% 0,76-0,92) (31).

En conclusión, en nuestro medio, la prevalencia es mayor a la estimada previamente; las nuevas definiciones y la reducción del punto de corte para caracterizar la obesidad abdominal hacen que se pueda diagnosticar esta patología en un mayor número de personas. La constelación de factores de riesgo, como la conocemos hoy, está asociada en estudios prospectivos al desarrollo de diabetes mellitus (si no está presente al momento de la inclusión del caso índice) y de enfermedad cerebrocardiovascular con las definiciones previamente utilizadas; se necesitan estudios que confirmen que, con las adiciones y modificaciones que se han hecho a la definición, se preserva su poder de predicción para estos dos desenlaces.

Una tercera parte de la población investigada, que en promedio no supera los 40 años, tiene características propias del síndrome; las variables más comúnmente observadas son la obesidad abdominal y la hipoalfalipoproteinemia. Es importante determinar los factores de riesgo asociados a tales características e ilustrar a la comunidad sobre las intervenciones que buscan modificar el estilo de vida, las cuales pueden tener un gran impacto sobre ambos componentes.

\section{Conflictos de interés}

No existen conflictos de interés.

Una versión resumida del trabajo fue presentada como afiche en el XXI Congreso Colombiano de Cardiología y Cirugía Cardiovascular, Cartagena, febrero de 2006.

\section{Financiación}

Esta investigación fue financiada por la Dirección General de Investigaciones de la Universidad Autónoma de Bucaramanga, proyecto EGEN 18.

\section{Referencias}

1. Bautista LE. Tendencias de la mortalidad por enfermedades cardiovasculares en Colombia y Santander, 1980-1996. Bucaramanga: Universidad Industrial de Santander, Organización Panamericana de la Salud; 2000.
2. Cubillos-Garzón LA, Casas JP, Morillo CA, Bautista LE. Congestive heart failure in Latin America: the next epidemic. Am Heart J. 2004;147:412-7.

3. Pearson TA. Cardiovascular disease in developing countries: myths, realities, and opportunities. Cardiovasc Drugs Ther. 1999;13:95-104.

4. Isomaa B, Almgren P, Tuomi T, Forsen B, Lahti K, Nissen M, et al. Cardiovascular morbidity and mortality associated with the metabolic syndrome. Diabetes Care. 2001;24:683-9.

5. Sattar N, Gaw A, Scherbakova O, Ford I, O'Reilly DS, Haffner SM, et al. Metabolic syndrome with and without C-reactive protein as a predictor of coronary heart disease and diabetes in the West of Scotland Coronary Prevention Study. Circulation. 2003; 108:414-9.

6. Ridker PM, Buring JE, Cook NR, Rifai N. C-reactive protein, the metabolic syndrome, and risk of incident cardiovascular events: an 8-year follow-up of 14719 initially healthy American women. Circulation. 2003;107:391-7.

7. Bautista LE, Morillo CA, Casas JP, Arenas IA, Ardila E. Estudio global de casos y controles para identificar factores de riesgo para infarto agudo del miocardio in diferentes poblaciones étnicas. INTERHEARTColombia. Reporte técnico. Bogotá D.C: Colciencias; 2002.

8. Haffner SM, D'Agostino R, Saad MF, Rewers M, Mykkanen L, Selby J, et al. Increased insulin resistance and insulin secretion in nondiabetic AfricanAmericans and Hispanics compared with non-hispanic whites. The Insulin Resistance Atherosclerosis Study. Diabetes. 1996;45:742-8.

9. Han TS, Sattar N, Williams K, Gonzalez-Villalpando C, Lean ME, Haffner SM. Prospective study of Creactive protein in relation to the development of diabetes and metabolic syndrome in the Mexico City Diabetes Study. Diabetes Care. 2002;25:2016-21.

10. Expert Panel on Detection, Evaluation, and Treatment of High Blood Cholesterol in Adults. Executive Summary of The Third Report of The National Cholesterol Education Program (NCEP) Expert Panel on Detection, Evaluation, and Treatment of High Blood Cholesterol in Adults (Adult Treatment Panel III). JAMA. 2001;285:2486-97.

11. Ford E, Giles W, Dietz W. Prevalence of the metabolic syndrome among US adults: findings from the third National Health and Nutrition Examination Survey. JAMA. 2002;287:356-9.

12. Aschner P. Diabetes trends in Latin America. Diabetes Metab Res Rev. 2002; 18 (Suppl 3):S27-31.

13. Villegas A, Botero LF. Prevalencia de síndrome metabólico en la población de El Retiro. Rev ALAD. 2004;12:20. 
14. Flórez H, Silva E, Fernández V, Ryder E, Sulbarán T, Campos G, et al. Prevalence and risk factors associated with the metabolic syndrome and dyslipidemia in White, Black, Amerindian and Mixed Hispanics in Zulia State, Venezuela. Diabetes Res Clin Pract. 2005;69:63-77.

15. Grundy SM, Cleeman JI, Daniels SR, Donato KA, Eckel RH, Franklin BA, et al. Diagnosis and management of the metabolic syndrome. An American Heart Association/National Heart, Lung, and Blood Institute Scientific Statement. Circulation. 2005;112:2735-52.

16. Alberti KG, Zimmet P, Shaw J. Metabolic syndrome a new world-wide definition. A Consensus Statement from the International Diabetes Federation. Diabet Med. 2006;23:469-80.

17. Pickering TG. For the American Society of Hypertension Ad Hoc Panel. Recommendations for the use of Home (Self) and Ambulatory Blood Pressure Monitoring. Am J Hypertens. 1996;9:1-12.

18. Marks GC, Habickt JP, Mueller WH. Reliability, dependability, and precision of anthropometric measurements, The Second National Health and Nutrition Examination Survey 1976-1980. Am J Epidemiol. 1989;130:578-87.

19. Wilcoxon F. Individual comparisons by ranking methods. Biometrics. 1945;1:80-3

20. Cohen J. A coefficient of agreement for nominal scales. Educ Psychol Measur. 1960;20:37-46.

21. StataCorp. Stata statistical software: release 9,2. College Station: StataCorp LP; 2005.

22. Kahn R, Buse J, Ferrannini E, Stern M, American Diabetes Association, European Association for the Study of Diabetes. The metabolic syndrome: Time for a critical appraisal: joint statement from the American Diabetes Association and the European Association for the Study of Diabetes. Diabetes Care. 2005;28:2289-304.

23. Matsuzawa Y, Funahashi T, Kihara S, Shimomura I. Adiponectin and metabolic syndrome. Arterioscler Thromb Vasc Biol 2004;24:29-33.
24. Karter AJ, D'Agostino RB Jr, Mayer-Davis EJ, Wagenknecht LE, Hanley AJ, Hamman RF, et al. Abdominal obesity predicts declining insulin sensitivity in nonobese diabetes. Obes Metabolism. 2005;7:230-8.

25. Perez M, Casas JP, Cubillos-Garzon LA, Serrano NC, Silva F, Morillo CA, et al. Using waist circumference as a screening tool to identify Colombian subjects at cardiovascular risk. Eur J Cardiovasc Prev Rehabil. 2003;10:328-35.

26. Lorenzo C, Serrano-Rios M, Martínez-Larrad MT, Gabriel R, Williams K, Gómez-Gerique JA, et al. Central adiposity determines prevalence differences of the metabolic syndrome. Obes Res 2003;11:1480-7.

27. Mendivil C, Sierra I, Pérez C. Valoración del riesgo cardiovascular global y prevalencia de dislipemias según los criterios del NCEP-ATPIII en una población adulta de Bogotá, Colombia. Clin Invest Arterioscl. 2004;16:99-107

28. Guerrero-Romero F, Rodríguez-Morán M. Concordance between the 2005 International Diabetes Federation Definition for Diagnosing Metabolic Syndrome With the National Cholesterol Education Program Adult Treatment Panel III and the World Health Organization definitions. Diabetes Care. 2005;28:2588-9.

29. Ford ES. Prevalence of the metabolic syndrome defined by the International Diabetes Federation among adults in the U.S. Diabetes Care. 2005;28:2745-9.

30. Adams RJ, Appleton S, Wilson DH, Taylor AW, Dal Grande E, Chittleborough C, et al. Population comparison of two clinical approaches to the metabolic syndrome implications of the new International Diabetes Federation consensus definition. Diabetes Care. 2005;28:2777-9

31. Carnethon MR, Loria CM, Hill JO, Sidney S, Savage P, Liu K. Risk factors for the metabolic syndrome: The Coronary Artery Risk Development in Young Adults (CARDIA) study, 1985-2001. Diabetes Care. 2004; 27:2707-15. 